\title{
Ordering dynamics of microscopic models with nonconserved order parameter of continuous symmetry
}

\section{Zhang, Z.; Mouritsen, Ole G.; Zuckermann, Martin J.}

Published in:

Physical Review E. Statistical, Nonlinear, and Soft Matter Physics

Link to article, DOI:

10.1103/PhysRevE.48.2842

Publication date:

1993

Document Version

Publisher's PDF, also known as Version of record

Link back to DTU Orbit

Citation (APA):

Zhang, Z., Mouritsen, O. G., \& Zuckermann, M. J. (1993). Ordering dynamics of microscopic models with nonconserved order parameter of continuous symmetry. Physical Review E. Statistical, Nonlinear, and Soft Matter Physics, 48(4), 2842-2849. https://doi.org/10.1103/PhysRevE.48.2842

\section{General rights}

Copyright and moral rights for the publications made accessible in the public portal are retained by the authors and/or other copyright owners and it is a condition of accessing publications that users recognise and abide by the legal requirements associated with these rights.

- Users may download and print one copy of any publication from the public portal for the purpose of private study or research.

- You may not further distribute the material or use it for any profit-making activity or commercial gain

- You may freely distribute the URL identifying the publication in the public portal 


\title{
Ordering dynamics of microscopic models with nonconserved order parameter of continuous symmetry
}

\author{
Z. Zhang \\ Centre for the Physics of Materials, Department of Physics, McGill University, Montréal, Québec, Canada H3A 2T8 \\ O. G. Mouritsen \\ Canadian Institute of Advanced Research and Department of Physical Chemistry, The Technical University of Denmark, Building 206, \\ DK-2800 Lyngby, Denmark \\ M. J. Zuckermann \\ Centre for the Physics of Materials, Department of Physics, McGill University, Montréal, Québec, Canada H3A 2T8
}

(Received 25 June 1993)

\begin{abstract}
Numerical Monte Carlo temperature-quenching experiments have been performed on two threedimensional classical lattice models with continuous ordering symmetry: the Lebwohl-Lasher model [Phys. Rev. A 6, 426 (1972)] and the ferromagnetic isotropic Heisenberg model. Both models describe a transition from a disordered phase to an orientationally ordered phase of continuous symmetry. The Lebwohl-Lasher model accounts for the orientational ordering properties of the nematic-isotropic transition in liquid crystals and the Heisenberg model for the ferromagnetic-paramagnetic transition in magnetic crystals. For both models, which have a nonconserved order parameter, it is found that the linear scale, $R(t)$, of the evolving order, following quenches to below the transition temperature, grows at late times in an effectively algebraic fashion, $R(t) \sim t^{n}$, with exponent values which are strongly temperature dependent and furthermore vary for different measures of the time-dependent length scale. The results are discussed in relation to modern theories of ordering dynamics in systems with continuous orderparameter symmetry.
\end{abstract}

PACS number(s): 61.30.-v, 64.60.Cn, 64.60.My, 75.10.Hk

\section{INTRODUCTION}

Our current understanding of the manner in which order develops dynamically in systems which are brought into far-from-equilibrium states by thermal quenching is based on the concept of universality [1-4]. Universality states that late-stage ordering dynamics can be grouped into a small number of universality classes characterized by the growth law which the ordering process obeys. Most often the growth law is algebraic,

$$
R(t) \sim t^{n},
$$

in time, $t$, where $R(t)$ is a measure of the time-dependent length scale which characterizes the coherence length of the evolving order. For some systems with built-in randomness, a logarithmic rather than an algebraic growth law is expected [5]. For ordering processes which have algebraic growth laws, two main universality classes have been suggested: one for nonconserved order parameter with $n=\frac{1}{2}$ and one for conserved order parameter with $n=\frac{1}{3}$. Within this picture, only the nature of the conservation law for the order parameter is important for determining the value of $n$, and all other system properties are irrelevant, such as the spatial dimensionality, details of the interaction potential, the temperature, as well as the number, $p$, of thermodynamically degenerate ordered domains. Specific examples of ordering processes with conserved order parameter include spinodal decomposition in binary $[6,7]$ and ternary $[8,9]$ systems and phase separation in systems with phases of different density $[10,11]$. Examples of processes with a nonconserved order parameter include various continuous ordering and order-disorder processes in systems where the degeneracy index $p$ order is controlled by the occurrence of sublattice ordering, e.g., in adsorbed layers on surfaces $[4,12]$. Although the concept of universality is not firmly established, and there is controversy as to the number of universality classes, there is an increasing amount of theoretical and experimental evidence which supports the basic picture outlined here [3].

In the brief description outlined above of our current understanding of the universality in ordering dynamics it was tacitly assumed that the ordering symmetry is discrete and determined by a number, $p$, of equivalent states, e.g., $p=2$ in the ferromagnetic Ising model which describes phase separation and spinodal decomposition in a binary mixture, and it was assumed that the value of $p$ is irrelevant for the universal classification. However, for ordering processes characterized by a continuous ordering symmetry this simple picture may break down. A possible physical reason for such a breakdown could be that, whereas the ordering process for discrete symmetry can be characterized in terms of the formation of welldefined domains bounded by a network of localized domain boundaries, in the case of continuous symmetry 
there is no clear concept of domain boundaries. In the classical phenomenological theories of Lifshitz-AllenCahn [1] and Lifshitz-Slyozov [13] the presence of localized domain boundaries are crucial for the general argument which leads to the two universality classes described by $n=\frac{1}{2}$ and $n=\frac{1}{3}$, respectively. Nevertheless, in the case of continuous symmetry it has been suggested [14] that the ordering process, despite the absence of sharply defined domain walls, can still be characterized by a correlation function, $C(r, t)$ and a time-dependent structure factor, $S(q, t)$, which obey the scaling forms [14]

$$
\begin{aligned}
& C(r, t)=f\left[r R^{-1}(t)\right], \\
& S(q, t)=R^{d}(t) g[q R(t)],
\end{aligned}
$$

where $x=q R(t)$ is a scaling variable, $f(x)$ and $g(x)$ are scaling functions, and $d$ is the spatial dimension. $R(t)$ is then an appropriate time-dependent measure of the coherence length of the evolving order.

Recently, substantial theoretical activity involving both analytical and numerical work has been directed towards a description of ordering processes in systems with continuous symmetry [7,14-26]. The analytical theories, which build on a renormalization-group approach and which are well developed in the case of a conserved order parameter $[14,16,27]$, suggest for order-parameter symmetries $\mathrm{O}(N)$ the existence of a separate universality class described by an algebraic growth law with exponent value $n=\frac{1}{4}$. The analytical result has been supported by numerical calculations on phenomenological models with Langevin dynamics [19,22]. The case of ordering dynamics for a nonconserved order parameter is less well developed $[14,17]$ but the available field-theory and renormalization-group calculations suggest that one should recover the Lifshitz-Allen-Cahn growth-exponent value $n=\frac{1}{2}$. Numerical work using cell-dynamics simulation on nonconserved models with order parameters of continuous symmetry lead to exponent values, $n \simeq 0.42-44$, which are slightly below the Lifshitz-AllenCahn value $[18,23-25]$.

There is no experimental data available for the dynamics of conserved order parameter with continuous symmetry but it has been suggested [22] that the roughening of crystal-vapor interfaces may be a candidate for a system in this class. Liquid-crystal systems, for which the nematic phase is formed through an ordering process of continuous symmetry with a nonconserved order parameter, have been studied experimentally via the structure factor [28,29] and a modified Porod law has been found to be consistent with the theoretical results [20]. There is, however, a problem in that the nematic ordering has a more general symmetry than $O(N)$ due to the lack of head-tail symmetry breaking.

So far no numerical work has been reported on ordering dynamics in systems with continuous orderparameter symmetry using microscopic models. In the present paper we report on the results of a computersimulation study, using statistical-mechanical microscopic lattice models, of the ordering dynamics in nonconserved systems with continuous symmetry. We shall re- turn to the case of conserved order parameter in a later publication [30]. By using computer-simulated temperature quenching on microscopic models, one does not have to use coarse-grained variables, and the effects of the quench temperature can be studied in a more natural way. Furthermore, different measures of the evolving order from the simulations can be derived in a straightforward manner.

We have studied two different three-dimensional models. The first model, on which the major part of the present paper is based, is the Lebwohl-Lasher model [31] of the orientational ordering process in the isotropicnematic phase transition in liquid crystals. The model has a nonconserved (nematic) order parameter of continuous symmetry which is not quite the true $O(3)$ symmetry due to the inversion symmetry of the nematic director. The second model is the three-dimensional classical ferromagnetic Heisenberg model which has the true $\mathrm{O}$ (3) symmetry. By deriving results for both of these models we are in a position to determine whether the difference in the type of continuous symmetry is pertinent for the ordering dynamics in the case of nonconserved order parameter.

In Secs. II and III we define the two models and describe the computational techniques used. The results are presented in Sec. IV, and the paper is concluded in Sec. $\mathrm{V}$ by a discussion of the results in relation to theoretical predictions and to the concept of universality in ordering dynamics.

\section{MODELS}

The three-dimensional Lebwohl-Lasher model is described by the Hamiltonian

$$
\mathscr{H}=-\epsilon \sum_{i, j} P_{2}\left(\cos \theta_{i j}\right),
$$

where $P_{2}\left(\cos \theta_{i j}\right)=\frac{1}{2}\left(3 \cos ^{2} \theta_{i j}-1\right), \theta_{i j}$ is the angle between the axes of rotor molecules at nearest-neighbor sites $i$ and $j$, and $\epsilon$ is a positive coupling parameter. The rotors are positioned on a simple cubic lattice. The Lebwohl-Lasher model [31] is the lattice version of the Maier-Saupe model of an anisotropic liquid in which the molecules are coupled by the Hamiltonian in Eq. (4). The Lebwohl-Lasher model is the canonical model of a system which displays an orientational phase transition and in this respect it plays a role in the field of liquid crystals similar to that played by the Ising model in the general field of order-disorder phenomena in alloys and magnetic systems. The Lebwohl-Lasher model, which neglects the translational variables and the steric effects of the rotor molecules, is known to produce a very weak first-order orientational phase transition at $k_{B} T_{c} / \epsilon=1.1232[32,33]$. The nematic order in the Lebwohl-Lasher model is characterized by a second-rank-tensor order parameter, $\mathbf{Q}$, and a macroscopic, three-component director which is not coupled to the lattice. Hence the nematic ordering is of continuous symmetry.

The three-dimensional classical ferromagnetic Heisenberg model is defined by the Hamiltonian

$$
\mathscr{H}=-J \sum_{i, j} \mathbf{S}_{i} \cdot \mathbf{S}_{j}
$$


where $\mathbf{S}_{i}=\left(S_{x i}, S_{y i}, S_{z i}\right)$ is a classical spin vector of unit length, and $J>0$. The spin variables are arrayed on a simple cubic lattice. The Heisenberg model has a second-order phase transition from a ferromagnetic phase to a paramagnetic phase at $k_{B} T_{c} / J=1.44$. The order parameter in the ferromagnetic phase is a macroscopic magnetic moment specified by a length (the magnetic order parameter) and a direction in space. The magnetization direction in the Heisenberg model is not coupled to the lattice. Hence, the ferromagnetic order has continuous symmetry.

The two microscopic models have similar symmetry properties, but while the spins in the magnetic model in Eq. (5) have a sense of direction, and hence the continuous symmetry is of type $\mathrm{O}(3)$, the rotor model in Eq. (4) has an additional symmetry element corresponding to inversion of the rotors.

\section{COMPUTATIONAL TECHNIQUES}

The ordering dynamics in the Lebwohl-Lasher model and in the Heisenberg model has been studied by conventional Monte Carlo temperature-quenching simulations $[4,12]$. Since Monte Carlo techniques imply the use of stochastic dynamics to bring the quenched system towards thermodynamic equilibrium, the choice of dynamics is important when the Monte Carlo simulation is subject to a dynamical interpretation. Since we are studying a situation with a nonconserved order parameter, we have chosen the simplest possible Glauber excitation mechanism which corresponds to single-site random reorientation of the rotor or spin variable. It should be remarked that this dynamics does not necessarily correspond to the real dynamics in a liquid crystal or in a magnetic system. Under equilibrium conditions the nematic order parameter in a liquid crystal is usually conserved, whereas the direction of the director is not [34]. In the magnetic system, the spins are angular momentum operators and both the order parameter (the magnetization) as well as the magnetization direction are conserved quantities. Under nonequilibrium conditions, the actual conservation laws and the details of the equations of motion will depend on how the system interacts with the environment.

The simulations were performed on simple cubic lattices of different sizes, $L^{d}$, and subject to periodic boundary conditions. The quenches were carried out by initiating the system in a high-temperature disordered phase $(T \sim \infty)$ and instantly quenching to a temperature below the corresponding transition temperature. Each type of quench was performed a large number of times (typically 5-100) using different initial states, and the results have been averaged over the independent quenches to provide proper ensemble averages. This is a necessary procedure since the ordering processes are not subject to selfaveraging [35]. However, compared to quench studies of systems with discrete symmetry we find that less averaging is needed in order to obtain reliable ensemble values, probably because of the larger degree of randomness in the initial state of a system with continuous variables. The time is measured in Monte Carlo steps per lattice site
(MCS/S).

The time-dependent ordering has been monitored in a number of different ways. First, we calculated the excess internal energy

$$
\Delta E(t)=E(t)-\langle E(T)\rangle,
$$

where $\langle E(T)\rangle$ is the equilibrium internal energy at the temperature to which the system is quenched. Second, we calculated the time-dependent static structure factor, $S(q, t)$, and various length scales, $l(t)$ and $k_{m}(t)$, derived as follows from the intensity of the structure factor $S(q=0, t)$

$$
l(t)=[S(q=0, t)]^{1 / d}
$$

and from its moments

$$
k_{m}(t)=\left[\sum_{q}^{\prime} q^{m} S(q, t) / \sum_{q}^{\prime} S(q, t)\right]^{-1 / m},
$$

where the primed sums in Eq. (8) are restricted by an ultraviolet cutoff. Without an explicit assumption about the shape of the structure factor it is not possible to relate in a simple way these measures of the length scale obtained in reciprocal space to length scales in real space [36].

In the case of the Heisenberg model an average structure factor can be obtained (assuming translational invariance) as

$$
S(q, t)=\frac{1}{3} \sum_{\delta=x, y, z}\left\langle L^{-d}\left|\sum_{\mathbf{r}} S_{\mathbf{r}} e^{i q \hat{\mathrm{e}}_{\delta} \cdot \mathbf{r}}\right|^{2}\right\rangle,
$$

where $\widehat{\mathrm{e}}_{\delta}, \delta=x, y, x$, is an orthonormal set of vectors in $\mathbf{q}$ space.

Since the order parameter in the Lebwohl-Lasher model is a tensor order parameter, the structure factor is defined in terms of a local tensor order parameter

$$
Q_{\alpha \beta}(\mathbf{r})=\frac{3}{2} u_{\alpha}(\mathbf{r}) u_{\beta}(\mathbf{r})-\frac{1}{2} \delta_{\alpha \beta},
$$

where $\alpha, \beta=x, y, z$ and $u_{\alpha}(\mathbf{r})$ is the $\alpha$ component of a unit vector, $\mathbf{u}(\mathbf{r})$, which specifies the orientation of the rotor molecular at position $\mathbf{r}$. The structure factor is then determined from the Fourier transforms

$$
\widetilde{Q}_{\alpha \beta}\left(q \widehat{\mathbf{e}}_{\delta}\right)=\sum_{\mathbf{r}} Q_{\alpha \beta}(\mathbf{r}) e^{i q \hat{\mathbf{e}}_{\delta} \cdot \mathbf{r}},
$$

as

$$
S(q, t)=\frac{1}{3} \sum_{\delta=x, y, z}\left\langle L^{-d} \sum_{\alpha, \beta=x, y, z} \widetilde{Q}_{\alpha \beta} \widetilde{Q}_{\beta \alpha}^{*}\right\rangle .
$$

We have calculated the circularly averaged analog of Eq. (12) and found that it leads to the same time dependence as the one averaged over the canonical axes only.

\section{RESULTS}

We shall mainly concentrate on results for the ordering dynamics in the Lebwohl-Lasher model and only present some selected results derived for the Heisenberg model for the purpose of comparison. 


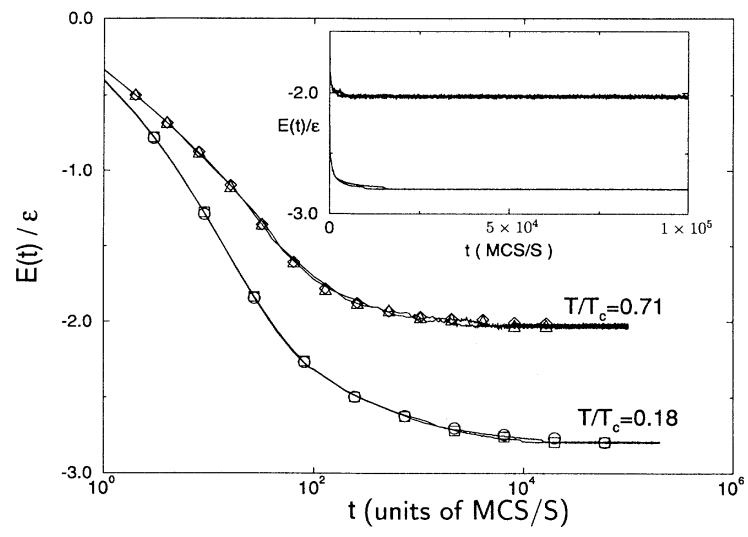

FIG. 1. Semilogarithmic plot of the development of the internal energy $E(t) / \epsilon$ as a function of time [ $t$ in units of Monte Carlo steps per site (MCS/S)] obtained from quenches of the Lebwohl-Lasher model from the isotropic, disordered phase to two different temperatures in the ordered, nematic phase. For each temperature the results of two different quenches are shown. The data refer to lattices with $28^{3}$ sites (solid lines) and $40^{3}$ sites (open symbols). The inset, which presents the same data for the small system in a normal plot, shows the approach to equilibrium over long times.

Figure 1 shows the evolution of the time-dependent internal energy $E(t) / \epsilon$ for the Lebwohl-Lasher model as obtained from quenches from the disordered phase to two different temperatures in the nematic phase. Results of two different quenches are shown for each temperature. The figure clearly shows that the equilibrium state can be reached within the time span of the simulation and that an accurate value of the equilibrium energy needed for Eq. (6) can be obtained. This should be contrasted to the usual result from deep quenches of lattice models with discrete symmetry, such as Ising and Potts models, where

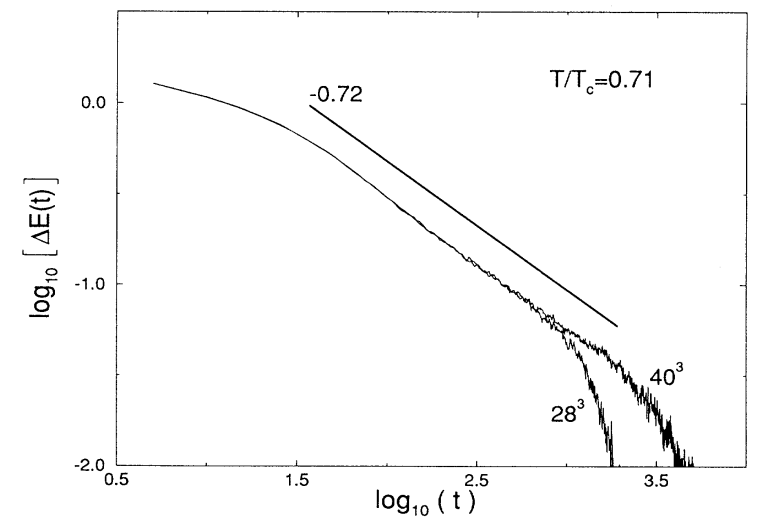

FIG. 2. Log-log plot of the excess energy, $\Delta E(t)$ in Eq. (6), vs time time ( $t$ in units of MCS/S) for quenches of the LebwohlLasher model from the disordered phase to a temperature, $T / T_{c}=0.71$ in the ordered phase. The results, which correspond to data obtained by averaging over 100 independent quenches, are shown for two different lattice sizes, $L^{d}=28^{3}$ (bottom curve) and $40^{3}$ (top curve). The solid line has a slope of -0.72 . the domain pattern often locks into a metastable slab configuration at late times [37].

Simulation data of the type shown in Fig. 1 is now analyzed in terms of a growth law as in Fig. 2 in case of the Lebwohl-Lasher model. The data shown illustrate the effects on the growth law due to the finite size of the system. It is seen that for the larger system the data conform to a power law, $\Delta E(t) \sim t^{-n}$, over almost two decades. A finite-size crossover effect is seen at long times and the crossover sets in earlier for smaller systems. The corresponding finite-size effects in the case of the length scales derived from the first and second moment of the structure factor are illustrated in Fig. 3. It is seen that both $k_{1}$ and $k_{2}$ grow effectively algebraically in time, but with different exponent values which are both considerably smaller than the growth exponent for the excess energy in Fig. 2.

The effect of the quench temperature on the ordering dynamics is analyzed in Fig. 4 and Fig. 5 in case of the Lebwohl-Lasher model and the Heisenberg model, respectively. For both models, a dramatic temperature dependence is observed. For each temperature, the data follow a power-law behavior over a substantial time range before the finite-size effects set in. As expected, the finite-size effects set in at an earlier time the higher the temperature is. This is because the amplitude of the relaxation rate is larger at higher temperatures. Hence we are able to obtain data which are not influenced by finitesize effects for a longer time span the lower the quench temperature is. For both models, the zero-temperature growth exponent for the excess energy is close to $n \simeq 0.25$ and it increases towards $n \simeq 1$ as the temperature is increased towards the respective transition-temperature value.

A comparison of the growth data for the different length-scale measures for the Lebwohl-Lasher model in the case of two different temperatures is shown in Fig. 6. The main conclusions to be drawn from this comparison

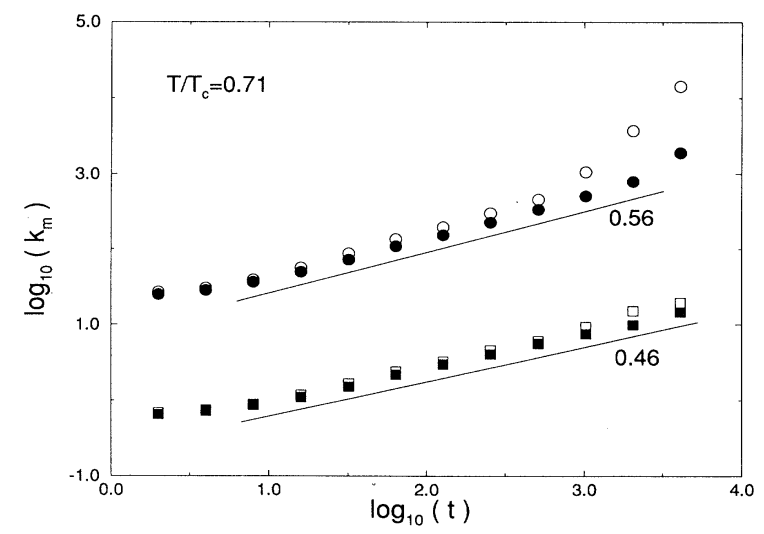

FIG. 3. Log-log plot vs time ( $t$ in units of MCS/S) of the length scales, $k_{1}(t)$ and $k_{2}(t)$ in Eq. (8), obtained using the first two moments of the structure factor in Eq. (12) for quenches of the Lebwohl-Lasher model from the disordered phase to a temperature, $T / T_{c}=0.71$ in the ordered phase. Results are shown for two different lattice sizes, $L^{d}=28^{3}$ (open symbols) and $40^{3}$ (solid symbols). The solid lines have slopes as indicated. 


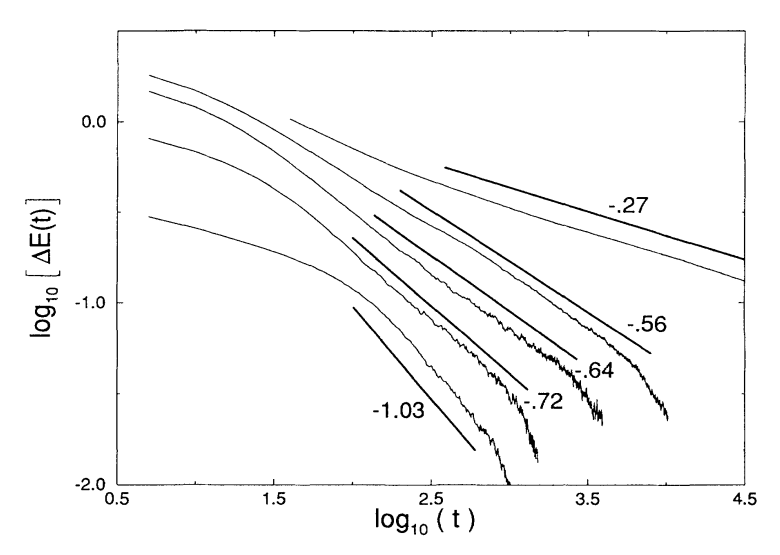

FIG. 4. Log-log plot of the excess energy, $\Delta E(t)$ in Eq. (6), vs time ( $t$ in units of MCS/S) for quenches of the Lebwohl-Lasher model from the disordered phase to different temperatures, $T / T_{c}$, in the ordered phase. From top to bottom the data sets correspond to temperatures $T / T_{c}=0,0.18,0.53,0.71$, and 0.89 . The data are obtained for a lattice with $L^{d}=28^{3}$ sites. For each set of data a solid line with a given slope is indicated.

are, as already noted, that there is a substantial increase in the effective growth exponent as the temperature is increased. Moreover, we conclude that the different length measures scale differently and the difference in their effective growth exponents is larger than the uncertainty for the time range and the lattice sizes studied. There is a clear tendency for the higher moments to lead to progressively lower growth exponents [we have also calculated $\left.k_{4}(t)\right]$. Furthermore, the length-scale measure derived from the structure-factor intensity at the Bragg point invariably has the lower exponent.

The same conclusions may be drawn from the data obtained from the Heisenberg model, some of which are shown in Fig. 7. There is a tendency for the Heisenberg model result to give growth-exponent values which vary more strongly with temperature compared to those of the

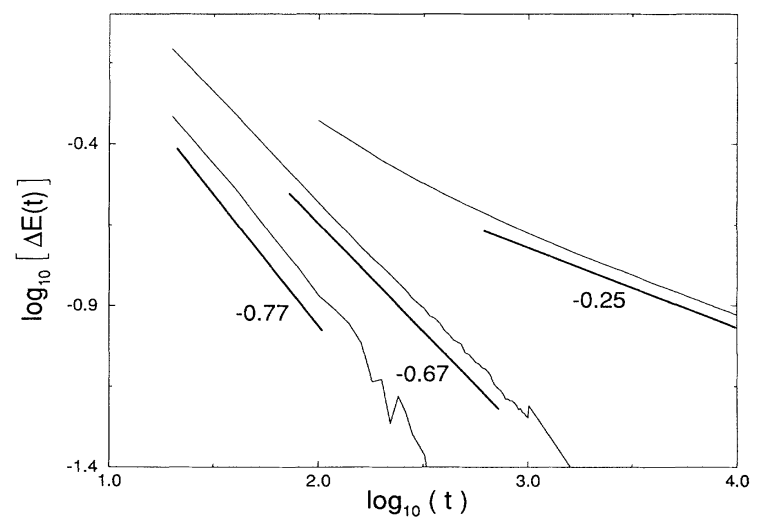

FIG. 5. Log-log plot of the excess energy, $\Delta E(t)$ in Eq. (6), vs time ( $t$ in units of MCS/S) for quenches of the Heisenberg model from the disordered phase to three different temperatures, $T / T_{c}$, in the ordered phase: (from top to bottom) $T / T_{c}=0$, $0.18,0.7$. The data are obtained for a lattice with $L^{d}=28^{3}$ sites. For each set of data a solid line with a given slope is indicated.

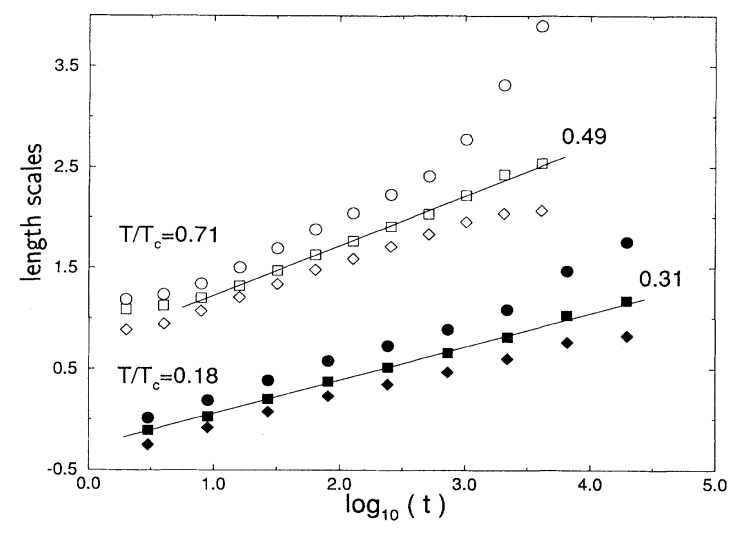

FIG. 6. Log-log plot vs time ( $t$ in units of MCS/S) of the length scales, $l(t)(\diamond), k_{1}(t)(\bigcirc)$, and $k_{2}(t)(\square)$ in Eqs. (7) and (8), obtained using the $q=0$ intensity and the first two moments of the structure factor in Eq. (12) for quenches of the LebwohlLasher model from the disordered phase to two different temperatures, $T / T_{c}=0.18$ and 0.71 , in the ordered phase. Results are shown for a lattice with $L^{d}=28^{3}$ sites. The solid lines have slopes as indicated.

Lebwohl-Lasher model. The combined results obtained from quenches to zero temperature are shown for the Heisenberg model in Fig. 8. We note that at zero temperature the different length-scale measures derived from the structure factor give similar exponent values which are lower than the value obtained from the excess energy, cf. Fig. 5. Results from quenches of the Lebwohl-Lasher model to zero temperature show the same systematics.

We have analyzed the high- $q$ tails of the structure factor of the two models in terms of a scaling function $S(q, t) / S(0, t)$ and a scaled variable $q S^{1 / d}(0, t)$ as shown in Fig. 9 in the case of the Lebwohl-Lasher model. Due to the small linear size of the systems simulated, data can

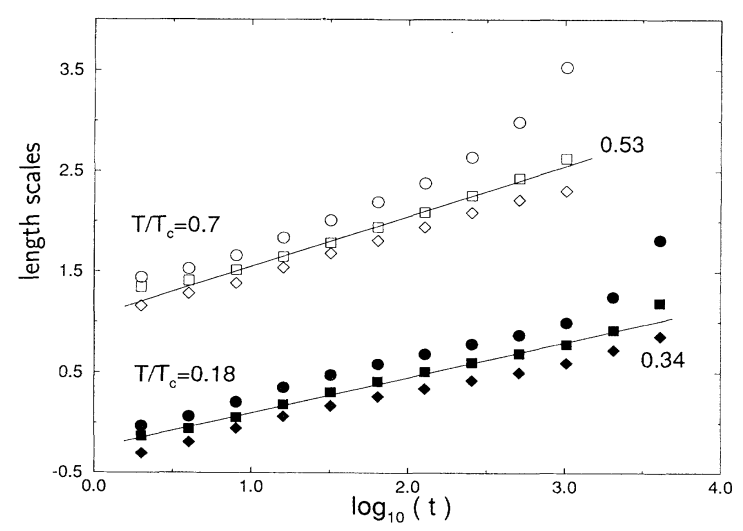

FIG. 7. Log-log plot vs time ( $t$ in units of MCS/S) of the length scales, $l(t)(\diamond), k_{1}(t)(\bigcirc)$, and $k_{2}(t)(\square)$ in Eqs. (7) and (8), obtained using the $q=0$ intensity and the first two moments of the structure factor in Eq. (9) for quenches of the Heisenberg model from the disordered phase to two different temperatures, $T / T_{c}=0.7$, and 0.18 , in the ordered phase. Results are shown for a lattice with $L^{d}=28^{3}$ sites. The solid lines have slopes as indicated. 


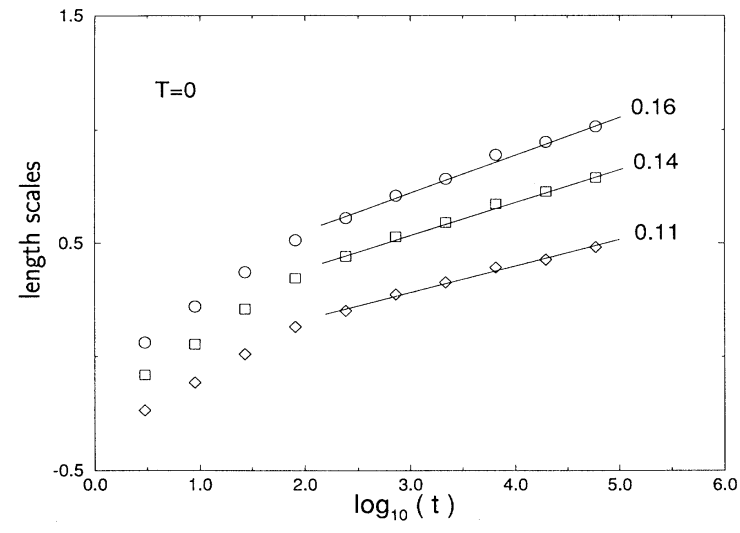

FIG. 8. Log-log plots vs time ( $t$ in units of MCS/S) of the length scales, $l(t)(\diamond), k_{1}(t)(\bigcirc)$, and $k_{2}(t)(\square)$ in Eqs. (7) and (8), obtained using the $q=0$ intensity and the first two moments of the structure factor in Eq. (9) for a quench of the Heisenberg model from the disordered phase to zero temperature, $T=0$. Results are shown for a lattice with $L^{d}=28^{3}$ sites. The solid lines have slopes as indicated.

only be obtained over a moderate range of $q$ values. The scaling of the data is not very clear but is, however, consistent with Porod-type law

$$
S(q, t) \sim q^{-\omega}
$$

with $\omega \simeq 5$. The data for the Heisenberg model show a similar appearance with the same value of $\omega$.

In order to shed more light on the microscopic phenomena which follow the ordering process in the Lebwohl-Lasher model, we have tried to identify ordered domains somewhat arbitrarily by locating those regions of the system which carry the excess internal energy at

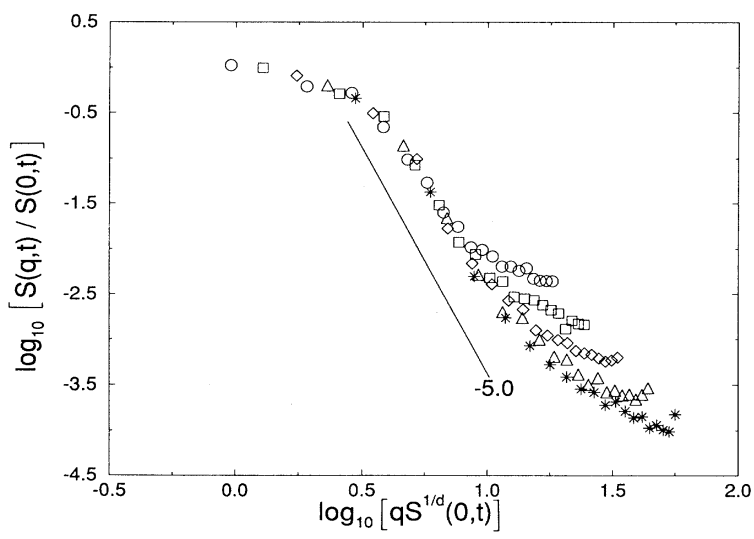

FIG. 9. Log-log plot of the scaled structure factor, $S(q, t) / S(0, t)$, vs the scaling variable, $q S^{1 / d}(0, t)$, for the Lebwohl-Lasher model. The data, which are obtained from a lattice with $L^{d}=40^{3}$ sites, are shown for a series of times (in units of MCS/S), $t=2^{6}(\bigcirc), 2^{7}(\square), 2^{8}(\triangle), 2^{9}(\diamond)$, and $2^{10}(*)$. The solid line has the slope -5 .

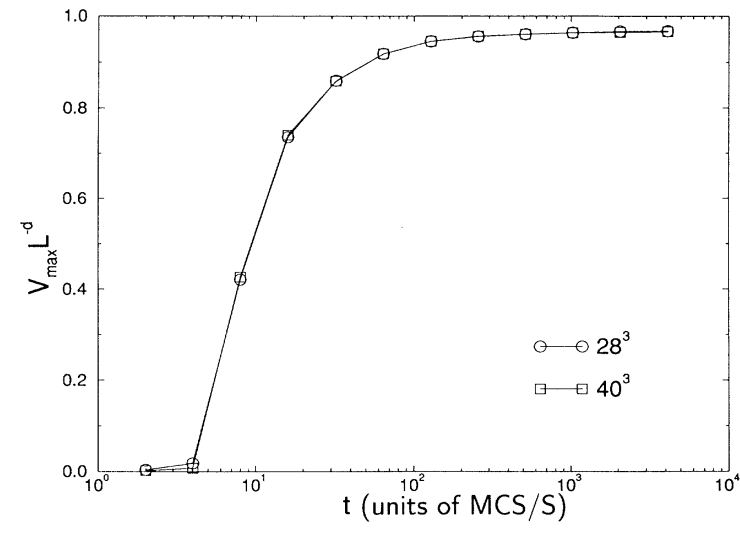

FIG. 10. Semilogarithmic plot of the time dependence (time $t$ in units of MCS/S) of the size, $V_{\max } L^{-d}$, of the normalized, largest ordered domain in the Lebwohl-Lasher model after a quench from the disordered phase to a temperature $T / T_{c}=0.71$ in the ordered phase. Results are shown for two different lattice sizes, $L^{d}=28^{3}(O)$ and $40^{3}(\square)$. The solid lines are guides to the eye.

any given time. For that purpose we have defined ordered domains as connected sets of bonds between pairs of rotors on the lattice which have interaction energies smaller or equal to the equilibrium energy per bond at the temperature in question. Using this definition we derived a normalized, largest ordered domain $V_{\max }(t) L^{-d}$. Simulation results for this quantity are shown in Fig. 10 for two different lattice sizes. It is seen that at very early times, $t \sim 100 \mathrm{MCS} / S$, a single domain has taken over the whole system and the concept of domains and domain wall loses its meaning. The fact, that the time at which the largest domain extends over the whole system does not depend on system size, shows that this is not a finitesize effect. The same results have been obtained for the Heisenberg model. Hence, the ordering process in these microscopic models with order parameters of continuous symmetry proceeds in a highly delocalized way involving the cooperative reorientation of large regions of the system. The use of the inverse excess energy, $\Delta E^{-1}(t)$, as a length-scale measure is therefore questionable since it assumes [12] a relation between the excess energy and a perimeter of a domain-wall network which is not present in these models.

\section{DISCUSSION}

We have in this paper presented the results of a comparative computer-simulation study of the dynamics of ordering in two microscopic statistical-mechanical lattice models with nonconserved order parameter of continuous symmetry. The main result of the study is that the timedependent excess energy, as well as the different measures of time-dependent length scale which characterize the evolving order after a quench from the disordered phase to the ordered phase, conform to effectively algebraic growth laws with values of the growth exponent which are different for the excess energy and different length- 
scale measures. Furthermore, the growth exponent for each length-scale measure is different from that of the others and it varies with temperature. An analysis of real-space configurations reveals that at late stages the ordering process cannot be characterized in terms of a well-defined domain-boundary network. Hence, the length-scale measure derived from the inverse excess energy does not behave like a proper length scale for our results, although it still monitors the time evolution of the ordering process in a global manner. Therefore, our results for the ordering dynamics in the two microscopic models studied do not conform to the usual ideas of dynamical scaling and universality.

The important question is: do the ordering dynamics in both microscopic models conform to the dynamical scaling behavior of Eqs. (2) and (3)? The renormalizationgroup analysis of Bray [14] is based on a coarse-grained Langevin equation and gives a clear picture of dynamical scaling for models with a conserved vector order parameter. Bray states that the case of domain growth for a nonconserved order parameter is more tenuous and proposes that a contribution from the elimination of short length-scale degrees of freedom should be included in the analysis. He argues convincingly that this may be described in terms of an intermediate length scale representing the thickness of interfaces and directly proportional to the surface tension. Under these circumstances Bray obtains a growth exponent $n=\frac{1}{2}$ corresponding to dynamical scaling for a nonconserved order parameter in the universality class of the Ising model for both scalar and vector order parameters. Direct simulation of the nonconserved Langevin equation for nematic liquid crystals gave a growth exponent $n=0.44$ in three dimensions [18]. It is most likely that our system sizes are such that we would still be in the intermediate scaling regime of Bray, in which case it is not unreasonable that our results do not conform to the dynamic scaling predictions and exhibit temperature-dependent effective growth exponents. It is interesting to note that the results for both our models are very similar, even though the Lebwohl-Lasher model has inversion symmetry.

Another important question relates to the nature and role of the defects in the ordering dynamics of systems with a nonconserved order parameter. Liu and Mazenko [17] invoke the presence of stable topological defects and propose that the corresponding defect dynamics controls both the bulk ordering field and short-distance behavior in the scaling regime. This point has been studied in detail by Bray and his co-workers $[20,38]$ in terms of the dependence of Porod's law on the nature of the defects. In particular the form of Porod's law is that the high- $q$ tail in the structure factor can be written as follows in the scaling regime:

$$
S(q, t) \sim q^{-(d+m)},
$$

$m=1$ for domain walls, $m=2$ for strings or vortices, and $m=3$ for monopoles. Bray et al. [20] obtain a value of $m=2$ for nematic liquid crystals. They also show that there is reasonable agreement with experiment for small enough values of $q$. Our data of Fig. 9 for the high- $q$ tail corresponding to the Lebwohl-Lasher model is consistent with this value even though the defects in our simulations cannot be identified as strings. However, Fig. 9 does show that, due to the sizes of our systems, the tail can only be analyzed over a very short range of $q$ values.

Furthermore, Bray [14] argues that temperature is an irrelevant variable and, under certain circumstances, a dangerously irrelevant variable in the scaling regime. In contrast our data show that the growth exponent for both the microscopic models considered here for the nonconserved case is strongly temperature dependent. This result supports our earlier conjecture regarding the role of intermediate length scales in dynamic scaling for systems with nonconserved vector order parameters.

To conclude, our results are not in agreement with the theoretical results based on field theory [17] and approximate-renormalization-group theory [14] which for the case of ordering dynamics in systems with nonconserved order parameter of continuous symmetry predicts that the classical Lifshitz-Allen-Cahn growth law with $n=\frac{1}{2}$ should be recovered. It is difficult, based on the present numerical work and the approximate nature of the current theory, to assess the reason for the discrepancies. It is interesting to note that a possible route to clarify this situation further may be to numerically study the effect of fields which break the continuous symmetry. Earlier work [39] on two-dimensional $X Y$ models with cubic anisotropy which breaks the continuous symmetry, introduced the concept of soft domain walls whose width could be varied with the strength of the symmetry-breaking field. In the region of "hard" and localized domain walls the well-known results for ordering dynamics with nonconserved order parameter were found, whereas when the softness was increased and the domain-wall thickness became large compared to the system size, the effective growth exponents increased beyond the hard-wall values.

\section{ACKNOWLEDGMENTS}

The work was supported by FCAR du Quebec under a centre and team grant, by NSERC of Canada, and by the Danish Natural Science Research Council under Grant No. 11-0065-1.
[1] J. D. Gunton, M. San Miguel, and P. S. Sahni, in Phase Transitions and Critical Phenomena, edited by C. Domb and J. L. Lebowitz (Academic, New York, 1983), Vol. 8, p. 267.

[2] H. Furukawa, Adv. Phys. 34, 703 (1985).
[3] For an experimental overview of the field of ordering dynamics, see the selection of papers in Dynamics of Ordering Processes in Condensed Matter, edited by S. Komura and H. Furukawa (Plenum, New York, 1988).

[4] O. G. Mouritsen, in Kinetics of Ordering and Growth at 
Surfaces, edited by M. G. Lagally (Plenum, New York, 1990), p. 1.

[5] G. Grinstein and J. F. Fernandez, Phys. Rev. B 29, 6389 (1984).

[6] P. W. Voorhees, J. Stat. Phys. 38, 231 (1985).

[7] A. J. Bray, Phys. Rev. Lett. 62, 2841 (1989).

[8] C. Jeppesen and O. G. Mouritsen, Phys. Rev. B 47, 14724 (1993).

[9] M. Laradji, O. G. Mouritsen, and S. Toxvaerd (unpublished).

[10] O. G. Mouritsen, P. J. Shah, and J. V. Andersen, Phys. Rev. B 42, 4506 (1990).

[11] W. W. Mullins and J. Viñals, Acta Metall. 37, 991 (1989).

[12] A. Sadiq and K. Binder, J. Stat. Phys. 35, 517 (1984).

[13] I. M. Lifshitz and V. V. Slyozov, J. Phys. Chem. Solids 19 , 35 (1961).

[14] A. J. Bray, Phys. Rev. B 41, 6724 (1990).

[15] A. J. Bray and S. Puri, Phys. Rev. Lett. 67, 2670 (1991).

[16] A. J. Bray and K. Humayun, Phys. Rev. Lett. 68, 1559 (1992).

[17] F. Liu and G. F. Mazenko, Phys. Rev. B 45, 6989 (1992).

[18] R. E. Blundell and A. J. Bray, Phys. Rev. A 46, R6154 (1992).

[19] M. Siegert and M. Rao, Phys. Rev. Lett. 70, 1956 (1993).

[20] A. J. Bray, S. Puri, R. E. Blundell, and A. M. Somoza, Phys. Rev. E 47, R2261 (1993).

[21] H. Toyoki, Phys. Rev. E 47, 2558 (1993).

[22] M. Mondello and N. Goldenfeld, Phys. Rev. E 47, 2384 (1993).

[23] M. Mondello and N. Goldenfeld, Phys. Rev. A 45, 657 (1992).
[24] M. Mondello and N. Goldenfeld, Phys. Rev. A 42, 5865 (1993).

[25] H. Toyoki, J. Phys. Soc. Jpn. 60, 1153 (1991); 60, 1433 (1991).

[26] A. Coniglio and M. Zannetti, Europhys. Lett. 10, 575 (1989).

[27] G. F. Mazenko and O. T. Valls, Phys. Rev. Lett. 59, 680 (1987).

[28] I. L. Chuang, N. Torok, and B. Yurke, Phys. Rev. Lett. 66, 2472 (1991); Science 251, 1336 (1991).

[29] A. P. Y. Wong, P. Wiltzius, and B. Yurke, Phys. Rev. Lett. 68, 3583 (1992).

[30] Z. Zhang (unpublished).

[31] P. A. Lebwohl and G. Lasher, Phys. Rev. A 6, 426 (1972); 7, 2222(E) (1973).

[32] U. Fabbri and C. Zannoni, Mol. Phys. 58, 763 (1986).

[33] Z. Zhang, O. G. Mouritsen, and M. J. Zuckermann, Phys. Rev. Lett. 69, 2803 (1992).

[34] P. G. De Gennes, The Physics of Liquid Crystals (Clarendon Press, Oxford, 1974).

[35] A. Milchev, K. Binder, and D. W. Heermann, Z. Phys. B 63, 521 (1986).

[36] J.-F. Willart, O. G. Mouritsen, J. Naudts, and M. Descamps, Phys. Rev. B 46, 8089 (1992).

[37] P. S. Sahni, G. Dee, J. D. Gunton, M. Phani, J. L. Lebowitz, and M. Kalos, Phys. Rev. B 24, 410 (1981).

[38] A. J. Bray, Phys. Rev. B 47, 228 (1993).

[39] O. G. Mouritsen, Phys. Rev. B 31, 2613 (1985); Phys. Rev. Lett. 56, 850 (1986); O. G. Mouritsen and E. Praestgaard, Phys. Rev. B 38, 2703 (1988). 